\title{
Marcadores sorológicos de hepatite B em in- divíduos submetidos a exames de sangue em unidades de saúde
}

Serological markers of hepatitis B in people submitted to blood testing in health care clinics

Lucia VG Miranda ${ }^{\mathrm{a}}$, Afonso DC Passos ${ }^{\mathrm{a}}$, José FC Figueiredo ${ }^{\mathrm{a}}$, Ana MC Gaspar ${ }^{\mathrm{b}}$ e Clara FT Yoshidab $^{b}$

a Faculdade de Medicina de Ribeirão Preto da Universidade de São Paulo. Ribeirão Preto, SP, Brasil. ${ }^{b}$ Laboratório de Referência Nacional para Hepatites Virais da Fundação Oswaldo Cruz. Rio de Janeiro, RJ, Brasil 


\title{
Marcadores sorológicos de hepatite $B$ em indivíduos submetidos a exames de sangue em unidades de saúde* Serological markers of hepatitis B in people submitted to blood testing in health care clinics
}

\author{
Lucia VG Miranda ${ }^{a}$, Afonso DC Passos ${ }^{\mathrm{a}}$, José FC Figueiredo ${ }^{\mathrm{a}}$, Ana MC Gaspar ${ }^{\mathrm{b}}$ e Clara \\ FT Yoshidab
}

aFaculdade de Medicina de Ribeirão Preto da Universidade de São Paulo. Ribeirão Preto, SP, Brasil. 'Laboratório de Referência Nacional para Hepatites Virais da Fundação O swaldo Cruz. Rio de Janeiro, RJ, Brasil

\section{Descritores}

Hepatite B, epidemiologia\#. Doadores de sangue ${ }^{\#}$. Fatores de risco. Hepatite $\mathrm{B}$, sangue.

\section{Keywords}

Hepatitis B,epidemiology". Blood donors ${ }^{\#}$. Risk factors. Hepatitis B, blood.

\section{Resumo}

\section{Objetivo}

Estudar aspectos da epidemiologia da hepatite B em pessoas submetidas à coleta de sangue em unidades de saúde.

Métodos

Indivíduos dos quais se coletou sangue em unidades de saúde de Ribeirão Preto, independentemente do motivo, foram solicitados a fornecer uma quantidade adicional de material, obtida no momento da coleta e submetida à detecção de marcadores de hepatite B. Simultaneamente, por meio de questionário padronizado, foram obtidas informações de possíveis fatores de risco para a doença. Os dados foram analisados por meio de um modelo de regressão logística.

\section{Resultados}

As prevalências de HBsAg e de anti-HBcAg foram de 0,3\% e 13,9\%, respectivamente. Os fatores de risco associados à infecção foram: idade, residência na cidade há menos de um ano, antecedente de hepatite, exposição prévia a casas de correção e homo/ bissexualismo masculino.

\section{Conclusões}

Devido a dificuldades crescentes de obtenção de sangue de indivíduos sadios, essa pode ser uma alternativa para estudos que objetivem fornecer informações sobre a circulação de agentes infecciosos na população. Embora não se possa generalizar os dados obtidos pela metodologia usada, ela traz conhecimento referente à circulação do vírus de hepatite $\mathrm{B}$.

\footnotetext{
Abstract

Objective

To study some of the epidemiological aspects of hepatitis $B$ in a non-representative sample of patients seen in health care clinics.

Methods

The study population comprised 632 patients who were seen at health care clinics in the city of Ribeirão Preto, Brazil, for the purpose of blood testing, regardless the reason. After signing a written consent, an additional amount of blood was drawn from the same venous puncture site used to collect the original sample for the testing
}

Correspondência para/Correspondence to: Afonso DC Passos

Av. Bandeirantes, 3.900

14049-900 Ribeirão Preto, SP, Brasil

E-mail: apassos@fmrp.usp.br
*Parte da dissertação de mestrado apresentada ao Departamento de Medicina Social da Faculdade de Medicina de Ribeirão Preto, USP, em 1997.

Edição subvencionada pela Fapesp (Processo n ${ }^{\circ}$ 00/01601-8).

Recebido em 26/1/1999. Reapresentado em 9/9/1999. Aprovado em 9/11/1999. 
assigned to the patient at the health care clinic. A questionnaire was applied to each participant, looking for the presence of risk factors for hepatitis $B$. The blood samples were tested for HBV markers, using immunoenzimatic techniques.

\section{Results}

The prevalences of $\mathrm{HBs} \mathrm{g}$ and anti-HBcAg were $0.3 \%$ and $13.9 \%$, respectively. By a logistic regression model, the following variables were significantly associated with the infection: age, time of residency in the city (higher risk among those living for a period less than one year), past history of hepatitis, incarceration and sexual behavior (higher risk among homosexual and bisexual males).

\section{Conclusions}

The growing difficulties in obtaining blood samples from a representative group of patients, as done in classic surveys, make it necessary to look for alternative methodologies which can provide information concerning the presence of infectious agents in a community. Though the results cannot be generalized to the population as a whole, the methodology used conveyed some knowledge regarding the circulation of hepatitis $B$ virus. In addition, it makes much easier to obtain agreement from the participants, since it does not add any invasive procedure. Despite the limitations, this methodology may be helpful in epidemiological surveillance of infectious agents known as producing asymptomatic infections in much of the population.

\section{INTRODUÇÃO}

Apesar de todos os recentes avanços em relação ao diagnóstico, ao tratamento e à profilaxia da hepatite $\mathrm{B}$, essa se mantém como um importante problema de saúde pública nos dias atuais. Particularmente relevante é o estudo da sua distribuição em diferentes populações humanas, uma vez que existem variações acentuadas na presença de marcadores segundo áreas e grupamentos distintos. ${ }^{12}$ Além disso, a busca do conhecimento da circulação viral representa uma atividade fundamental em vigilância epidemiológica, pois permite definir grupos de risco e orientar as estratégias de controle. ${ }^{13}$

Os estudos epidemiológicos, abordando a questão da distribuição da hepatite B em populações, são pouco freqüentes e geralmente limitam-se a grupos específicos, como doadores de sangue ${ }^{12}$ e gestantes. ${ }^{7}$ Isso é parcialmente causado pela dificuldade crescente de se executarem pesquisas, envolvendo coletas de sangue, em amostras representativas da comunidade, o que torna aconselhável a busca de novas metodologias que possam ser mais facilmente aceitáveis pela população.

O presente trabalho utiliza uma alternativa metodológica aos clássicos inquéritos populacionais, à medida que objetiva a determinação da prevalência de marcadores de hepatite $\mathrm{B}$ e de fatores de risco para essa doença em pacientes que, por qualquer razão, já estivessem se submetendo à coleta de sangue em unidades de saúde. Embora perdendo em representatividade, tal alternativa tem o mérito de contornar as dificuldades associadas à coleta de sangue, permitindo a monitorização da circulação do agente viral em segmentos da população.

\section{MÉTODOS}

O presente trabalho representa parte de uma investigação ampla sobre distribuição de marcadores de hepatites causadas pelos vírus $\mathrm{A}, \mathrm{B}$ e $\mathrm{C}$ e fatores de risco correspondentes, levada a efeito em unidades de saúde de Ribeirão Preto, São Paulo.

A população de estudo e de referência foi constituída por indivíduos que procuraram as 5 unidades distritais de saúde (UBDS) da cidade de Ribeirão Preto, entre outubro de 1994 e setembro de 1995, com vistas a serem submetidos à coleta de sangue, independentemente da razão que motivou a solicitação da coleta.

O tamanho amostral foi definido a partir da fórmula: $\mathrm{n}=\mathrm{Z}^{2} \mathrm{PQ} / \mathrm{d}^{2}{ }^{2}$ sendo $\mathrm{n}=$ tamanho amostral mínimo; $\mathrm{Z}=$ variável reduzida, cujo valor adotado foi de 1,96 para um alfa de 0,$05 ; \mathrm{P}=$ probabilidade de encontrar o fenômeno estudado. A inexistência de estimativas de prevalência de marcadores virais de hepatite na população de estudo levou à adoção de um valor igual a $50 \%: \mathrm{Q}=1-\mathrm{P} ; \mathrm{d}=$ precisão desejada, cujo valor adotado foi de $4 \%$.

Com base nesses critérios, o tamanho amostral mínimo foi definido como 600. Ao final do estudo, 632 indivíduos foram incluídos na amostra, distribuídos proporcionalmente à população estimada da área de abrangência de cada uma das 5 UBDS existentes em Ribeirão Preto. 


\section{Critérios de inclusão}

Para serem incluídos no estudo, os indivíduos deveriam satisfazer as seguintes condições: (1) ter idade igual ou superior a 1 ano e (2) concordar em participar da investigação e assinar um termo de autorização após tomar conhecimento dos objetivos e da metodologia do estudo. Menores de 13 anos só foram incluídos mediante termo assinado pelo responsável.

A realização do trabalho se deu entre outubro de 1994 e setembro de 1995. Cada uma das cinco UBDS foi visitada, diariamente, pelo período de tempo suficiente para se conseguir o tamanho amostral definido. As visitas ocorriam no período da manhã, quando os pacientes presentes para retirada de sangue eram reunidos para uma palestra rápida, abordando aspectos relevantes da epidemiologia da hepatite $B$. Nessa ocasião, oferecia-se a oportunidade de participação no trabalho, garantindo confidencialidade e retorno dos resultados sorológicos, bem como o acompanhamento médico especializado, para aqueles cujos resultados indicassem essa necessidade.

\section{Obtenção das amostras}

Utilizando-se a mesma punção usada para a coleta que motivou a ida à unidade de saúde, era obtida de cada participante uma amostra adicional de $7 \mathrm{ml}$ de sangue, coletada em tubo vacutainer. Após a centrifugação, o soro resultante era estocado à temperatura de $-20^{\circ} \mathrm{C}$ e enviado para análise laboratorial.

\section{Obtenção das informações individuais}

Na mesma ocasião da coleta da alíquota de sangue, cada participante respondia a um questionário padronizado com o objetivo de caracterizar a amostra quanto aos aspectos demográficos e socioeconômicos, além de detectar possíveis fatores de risco para hepatites virais. O questionário era aplicado por uma única pessoa aos indivíduos com idade igual ou superior a treze anos. Nos casos de menores de 13 anos ou de pacientes sem condições de fornecer respostas confiáveis (portadores de distúrbios mentais ou com limitações de comunicação), o questionário era respondido pelo acompanhante do paciente.

\section{Análise laboratorial do sangue}

Todas as amostras foram submetidas à detecção de marcadores sorológicos do vírus da hepatite $\mathrm{B}$ (HBsAg, anti-HBsAg e anti-HBcAg IgG), empregando-se testes imunoenzimáticos - Elisa. A pesquisa de HBsAg e anti-HBcAg foi realizada no Centro de Referência Nacional para Hepatites Virais (CRNHV) da
Fundação Oswaldo Cruz (Rio de Janeiro), utilizando reagentes preparados e padronizados pelo próprio centro, segundo metodologia e análises adotadas pelo mesmo. A pesquisa de anti-HBsAg foi realizada no Laboratório de Sorologia do Hospital das Clínicas de Ribeirão Preto (HCRP), utilizando reativo comercial (AUSAB EIA $^{\circledR}$ ) e seguindo metodologia e análise recomendadas pelo fabricante. As amostras positivas para o $\mathrm{HBsAg}$ foram submetidas à detecção do $\mathrm{HBe} A g$ e do anti-HBeAg, utilizando reativos comerciais (Imx $\mathrm{HBe} 2^{\circledR}$ e Imx anti-HBe ${ }^{\circledR}$ ), de acordo com metodologia e análise recomendadas pelo fabricante.

Para fins de análise, consideraram-se como positivas as amostras reagentes ao antígeno $\mathrm{HBsAg}$ e/ou ao anticorpo anti-HBcAg.

\section{Análise estatística}

Os dados foram submetidos a uma análise univariada, testando-se a existência de associação entre possíveis variáveis independentes (fatores de risco) e a presença de marcadores sorológicos. Isso se fez por meio do uso dos seguintes testes: qui-quadrado, qui-quadrado para tendências e teste exato de Fisher. As variáveis que demonstraram um valor de $\mathrm{p}<0,20$ foram incluídas em um modelo de regressão logística não condicional, ${ }^{9}$ tendo sido previamente testadas para a ocorrência de interação. Todos os procedimentos analíticos foram feitos com a utilização do software Stata 5.0.

\section{RESULTADOS}

Pela Tabela 1 observa-se que dos 632 indivíduos incluídos no estudo, houve predomínio do sexo feminino $(71,7 \%)$, que se manifestou em praticamente todas as faixas etárias. Ocorreu maior concentração de pessoas de idade mais elevada, particularmente acima de $40 \operatorname{anos} ; \chi^{2}(7 \mathrm{GL})=28,57(\mathrm{p}=0,000)$.

Tabela 1 - Distribuição da população estudada segundo sexo e faixa etária.

\begin{tabular}{lrrrrrr}
\hline Idade & \multicolumn{6}{c}{ Sexo } \\
\cline { 2 - 7 } & \multicolumn{2}{c}{ Masculino } & \multicolumn{2}{c}{ Feminino } & \multicolumn{2}{c}{ Total } \\
\cline { 2 - 7 } & $\mathrm{n}$ & $\%$ & $\mathrm{n}$ & $\%$ & $\mathrm{n}$ & $\%$ \\
\hline $1-4$ & 10 & 5,6 & 12 & 2,6 & 22 & 3,5 \\
$5-9$ & 8 & 4,5 & 10 & 2,2 & 18 & 2,8 \\
$10-19$ & 18 & 10,1 & 18 & 4,0 & 36 & 5,7 \\
$20-29$ & 11 & 6,1 & 64 & 14,1 & 75 & 11,9 \\
$30-39$ & 23 & 12,8 & 63 & 13,9 & 86 & 13,6 \\
$40-49$ & 34 & 19,0 & 67 & 14,8 & 101 & 16,0 \\
$50-59$ & 24 & 13,4 & 104 & 23,0 & 128 & 20,3 \\
$60 \mathrm{e}+$ & 51 & 28,5 & 113 & 24,9 & 164 & 25,9 \\
Ignor. & 0 & 0,0 & 2 & 0,4 & 2 & 0,3 \\
\hline Total & 179 & 100,0 & 453 & 100,0 & 632 & 100,0 \\
\hline
\end{tabular}


A Tabela 2 mostra a sorologia para o HBV segundo a idade, com a categoria "positivo" englobando todos os participantes com sorologia positiva para o $\mathrm{HBsAg}$ e/ou anti-HBcAg. A prevalência total foi de $13,9 \%$, com uma tendência de aumento proporcional à idade $\left(\chi^{2}\right.$ para tendência $\left.=14,54 ; p=0,000\right)$.

Tabela 2 - Distribuição dos marcadores sorológicos do vírus da hepatite B segundo a idade.

\begin{tabular}{|c|c|c|c|c|c|c|}
\hline \multirow[t]{3}{*}{ Idade } & \multicolumn{4}{|c|}{ Sorologia } & & \\
\hline & \multicolumn{2}{|c|}{ Positiva } & \multicolumn{2}{|c|}{ Negativa } & \multicolumn{2}{|c|}{ Total } \\
\hline & $n$ & $\%$ & $\mathrm{n}$ & $\%$ & $\mathrm{n}$ & $\%$ \\
\hline $1-4$ & 1 & 4,5 & 21 & 95,5 & 22 & 100,0 \\
\hline $5-9$ & 1 & 5,6 & 17 & 94,4 & 18 & 100,0 \\
\hline $10-19$ & 0 & 0,0 & 36 & 100,0 & 36 & 100,0 \\
\hline $20-29$ & 10 & 13,3 & 65 & 86,7 & 75 & 100,0 \\
\hline $30-39$ & 8 & 9,3 & 78 & 90,7 & 86 & 100,0 \\
\hline $40-49$ & 11 & 10,9 & 90 & 89,1 & 101 & 100,0 \\
\hline $50-59$ & 23 & 18,0 & 105 & 82,0 & 128 & 100,0 \\
\hline $60 e+$ & 34 & 20,7 & 130 & 79,3 & 164 & 100,0 \\
\hline Ignor. & 0 & 0,0 & 2 & 100,0 & 2 & 100,0 \\
\hline Total & 88 & 13,9 & 544 & 86,1 & 632 & 100,0 \\
\hline
\end{tabular}

Embora não considerada para efeitos de análise, a prevalência de anti-HBsAg foi igual a $15 \%$. Concomitância entre anti-HBcAg e anti-HBsAg ocorreu em 75 indivíduos. Em 19 pessoas verificou-se apenas presença de anti-HBsAg e em 13 a presença isolada de anti-HBcAg. Positividade para o marcador HBsAg aconteceu em apenas 2 indivíduos, ambos no grupo de idade igual ou superior a 60 anos, concomitantemente com a presença de anti-HBcAg.

$\mathrm{Na}$ análise univariada foram observadas associações estatísticas $(p<0,05)$ entre a distribuição de marcadores sorológicos e as seguintes variáveis: escolaridade (prevalências inversamente proporcionais ao grau da mesma); antecedente de hepatite; antecedente de hospitalização; preferência sexual (prevalência mais elevada entre homo e bissexuais masculinos); exposição a tratamentos dentários invasivos (prevalências mais elevadas em indivíduos com antecedente de cirurgias e/ ou extrações múltiplas); contato com casa de correção; tempo de residência em Ribeirão Preto (valores mais elevados para aqueles com menos de um ano).

Valores de "p" entre 5\% e 10\% foram observados para as variáveis: área de nascimento (maior prevalência em área rural quando cotejada com a urbana) e história de exposição prévia a unidades de diálise). Um valor de "p" entre $10 \%$ e $20 \%$ foi observado para a variável exposição profissional prévia ou atual a hospitais e serviços de saúde.

Todas as variáveis mencionadas, juntamente com a variável sexo, foram inicialmente submetidas à aná- lise multivariada pela aplicação de um modelo de regressão logística não condicional, segundo a apresentação mostrada na Tabela 3.

Tabela 3 - Variáveis selecionadas para a análise de regressão logística e tipo de apresentação das mesmas.

\begin{tabular}{|c|c|}
\hline Variável & Apresentação \\
\hline Idade & contínua \\
\hline Sexo & masc. $=1 ;$ femin.$=0$ \\
\hline Escolaridade & $\begin{array}{l}\text { nenhuma }=2 ; \text { primeiro e } \\
\text { segundo graus }=1 ; \\
\text { universitario }=0\end{array}$ \\
\hline Área de nascimento & rural $=1 ;$ urbana $=0$ \\
\hline Tempo de residência & $<1$ ano $=1 ;>1$ ano $=0$ \\
\hline Antec. de hepatite & $\operatorname{sim}=1 ;$ não $=0$ \\
\hline Trab. hosp. unid. saúde & $\operatorname{sim}=1 ;$ não $=0$ \\
\hline Antec. hospitalização & $\operatorname{sim}=1 ;$ não $=0$ \\
\hline Tratamento dentário & $\begin{array}{l}\text { cirurgia/extrações } \\
\text { múltiplas }=1 \cdot \text { outros }=0\end{array}$ \\
\hline $\begin{array}{l}\text { Unidade Diálise/Tx Renal } \\
\text { Casa de correção } \\
\text { Preferência sexual }\end{array}$ & $\begin{array}{l}\operatorname{sim}=1 ; \text { não }=0 \\
\operatorname{sim}=1 ; \text { não }=0 \\
\text { homo } / \text { bi }=1 ; \text { hetero }=0\end{array}$ \\
\hline
\end{tabular}

Após o controle dos fatores causadores de confusão, as seguintes variáveis apresentaram um valor de "p" menor do que 0,05: idade, tempo de residência em Ribeirão Preto, antecedente de hepatite e antecedente de contato com casa de correção. As variáveis antecedente de trabalho em hospitais/unidades de saúde e preferência sexual mostraram valores de probabilidade próximos ao limite de significância $(0,069$ e 0,055 , respectivamente), razão pela qual também foram incluídas no modelo final de regressão logística. A Tabela 4 permite ver que as variáveis, com exceção do antecedente de trabalho em hospitais/unidades de saúde, mantiveram associação com a presença de marcadores sorológicos na população estudada.

Tabela 4 - Resultado da análise do modelo final de regressão logística.

\begin{tabular}{lcrr}
\hline Variável & Odds Ratio & IC 95\% & $P$ \\
\hline Idade & 1,03 & $1,02-1,05$ & 0,000 \\
Tempo de residência & 3,54 & $1,60-7,85$ & 0,002 \\
Anteced. hepatite & 5,13 & $2,09-12,55$ & 0,000 \\
Trab. setor de saúde & 1,71 & $0,85-3,43$ & 0,133 \\
Casa de correção & 3,73 & $1,51-9,23$ & 0,004 \\
Pref. sexual & 5,71 & $1,20-27,08$ & 0,028 \\
\hline
\end{tabular}

\section{DISCUSSÃO}

Os empecilhos progressivamente maiores para se conseguir adesão a estudos, que envolvam coleta de amostras de sangue, dificultam cada vez mais a realização de inquéritos em amostras representativas da população como um todo. Tal situação representa problema relevante do ponto de vista da vigilância epidemiológica, à medida que dificulta o monitoramento da circulação de agentes causadores de infecções que nem sempre se exteriorizam clinicamente em grande número. Esse é o caso das hepatites virais que, por produzirem apreciável contingente de infecções assintomáticas, acabam por se 
disseminar na coletividade de uma maneira mais intensa que a evidenciada pelo aparecimento de casos clínicos. ${ }^{13}$

A metodologia utilizada no presente trabalho pretende ser uma alternativa que permita fornecer algum tipo de informação sobre a distribuição das hepatites virais em uma comunidade, sem que se utilizem amostras representativas da mesma. Embora perdendo no aspecto de generalização externa, a mesma apresenta a vantagem de contornar a dificuldade representada pela baixa adesão das pessoas aos inquéritos tradicionais, quando o procedimento a ser utilizado envolve a coleta de sangue em indivíduos aleatoriamente escolhidos para um determinado estudo. Ao fazer da sua população de estudo aqueles que se dirigiam à uma unidade de saúde para coleta de sangue, independentemente da razão que motivou a solicitação do exame, reduz-se em muito a relutância em participar da investigação, uma vez que o procedimento mais agressivo já seria, de qualquer maneira, utilizado.

A maior concentração do sexo feminino entre os participantes do estudo (Tabela 1) reflete, muito provavelmente, o padrão de utilização das unidades de saúde, com as mulheres se fazendo presentes de modo mais intenso que os homens. ${ }^{3,10}$

O valor de $15 \%$ na prevalência do marcador antiHBsAg, levemente superior ao valor mostrado pelo antiHBcAg (13,9\%), constituiu achado não esperado, uma vez que os dados da literatura apontam sistematicamente para valores mais elevados do anticorpo anti-HBcAg em estudos abrangendo amostras populacionais. ${ }^{4,8,16} \mathrm{No}$ presente estudo, se por um lado não pairam dúvidas sobre o passado de infecção nos 75 indivíduos que apresentaram positividade simultânea ao anti-HBcAg e ao anti-HBsAg, o mesmo não pode ser dito a respeito dos 19 em que apenas o anti-HBsAg se fez presente, situação em que não pode ser descartada a possibilidade de, pelo menos em alguns casos, a presença do marcador ser devida à vacinação prévia. Tal possibilidade deve ser mencionada, mesmo tendo-se em conta que a vacina era de uso muito pouco freqüente à época da realização da presente investigação, limitando-se a segmentos populacionais reduzidos. Como o questionário aplicado não contemplou a questão da imunização prévia, optou-se por excluir da análise os 19 indivíduos que apresentaram positividade exclusiva ao anti-HBsAg.

O valor de 13,9\% para a prevalência de marcadores sorológicos, bem como a presença de apenas 2 positivos para o HBsAg (0,32\%), ambos acima de 60 anos de idade, evidenciam o baixo nível de circulação do vírus B na população estudada. Particularizando para o HBsAg, a prevalência obtida é praticamente idêntica à verificada em doadores de sangue do Hemocentro de Ribeirão Pre- to* e um pouco inferior à observada em gestantes atendidas no Hospital das Clínicas da Faculdade de Medicina de Ribeirão Preto. ${ }^{7}$ A elevação da prevalência de acordo com a idade (Tabela 2) confirma o padrão de área de baixa circulação viral, onde a raridade ou inexistência de transmissão vertical ocasiona uma distribuição etária característica, com uma positividade que se revela escassa em idades precoces e tende a uma elevação lenta e gradual à medida que aumenta a faixa etária. ${ }^{1,6}$

$\mathrm{O}$ aumento do risco, associado ao fato de residir em Ribeirão Preto há menos de um ano, quando comparado a períodos maiores, possivelmente denota a relevância dos movimentos migratórios recentes e da urbanização na epidemiologia da hepatite $\mathrm{B}$, traduzindo as condições sociais precárias daqueles que são forçados a procurar uma nova alternativa de vida em outra cidade ou região. Tal fato foi bem demonstrado em investigação conduzida numa comunidade rural distante apenas $80 \mathrm{~km}$ de Ribeirão Preto, onde ficou evidente a associação de movimentos migratórios e positividade para hepatite B. Tal fato chama a atenção para a possibilidade da condição de migrante ser responsável por valores diferentes nas prevalências de marcadores da infecção por hepatite B, em áreas aparentemente semelhantes quanto às demais características. ${ }^{17}$

A associação com o antecedente de hepatite clinicamente diagnosticada, tal como verificada na presente investigação, não é um fato unanimemente apontado na literatura. Assim, a não-associação, quando descrita, tem sido explicada pelo fato de que cerca de $50 \%$ a $90 \%$ dos casos de hepatite B apresentam a doença de forma subclínica, o que conduz à ausência de diagnóstico em altas proporções. ${ }^{15}$ Outra possível explicação seria a impossibilidade de distinção clínica entre infecções causadas por vírus diferentes, fazendo com que casos de hepatite tipo A, ocorridos no passado, sejam recordados e referidos genericamente, como se o termo hepatite assumisse caráter designativo de uma doença única. ${ }^{16}$ Todavia, outros estudos têm mostrado associação entre antecedente de hepatite e marcadores do vírus B. ${ }^{11}$ Ainda hoje a história pregressa de hepatite viral após os 10 anos de idade constitui um dos critérios preconizados pelo Ministério da Saúde do Brasil para rejeição de potenciais doadores de sangue. ${ }^{14}$

Vários comportamentos de risco para a transmissão do vírus B podem ser detectados entre indivíduos expostos a prisões e/ou casas de correção, incluindo o uso endovenoso de drogas ilícitas, tatuagem com equipamentos não adequadamente esterilizados e relações sexuais desprotegidas, inclusive contatos homossexuais masculinos. ${ }^{5}$ No presente estudo, tal associação foi verificada com o antecedente de exposição a casas de 
correção, porém não com o antecedente de exposição a prisões, muito embora os números reduzidos de pessoas em ambas as categorias represente uma dificuldade para a interpretação adequada dos dados.

Positividade para hepatite B foi verificada nos dois únicos indivíduos que se identificaram como homossexuais masculinos e em dois dentre os seis que referiram bissexualidade. Esses baixos números justificam a grande amplitude do intervalo de confiança (Tabela 4), muito embora a prevalência observada nesses dois grupos, quando considerados em conjunto (50\%), tenha sido significativamente mais elevada que entre os heterossexuais.

Após a aplicação do modelo de regressão logística, desapareceu a associação entre marcadores de hepatite $B$ e atividade profissional no setor saúde. Tal associação mantinha-se no modelo multivariado quando se incluíam os 19 indivíduos positivos apenas para o anti-HBs. Esse fato favorece a hipótese de que, ao menos em parte, essa positividade possa ter sido resultante de vacinação prévia, mais factível de ter ocorrido entre alguns trabalhadores da área da saúde, numa época anterior à disponibilidade da vacina para outros segmentos populacionais. Justifica-se assim a não inclusão daqueles 19 indivíduos nas análises do presente estudo.

Embora os presentes dados não possam ser generalizados para a população de Ribeirão Preto, eles são úteis para a vigilância da situação do vírus da hepatite B. A repetição de estudos semelhantes, em períodos variados no futuro, poderá fornecer uma imagem mais acurada da circulação desse agente na comunidade. Por fim, os valores de prevalência encontrados são muito próximos dos observados na rotina do Hemocentro de Ribeirão Preto, sugerindo que a metodologia utilizada no presente estudo possa fornecer resultados comparáveis aos obtidos em indivíduos aparentemente normais que se apresentam para doação de sangue.

\section{REFERÊNCIAS}

1. Alter MJ, Hadler SC, Margolis HS, Alexander WJ, Hu PY, Judson $\mathrm{FN}$ et al. The changing epidemiology of hepatitis B in the United States. JAMA 1990;263:1218-22.

2. Berquó ES, Souza JMP, Gotlieb SLD. Bioestatística. São Paulo: EPU; 1981.

3. Brito FF, Marcelino IV, Forster AC, Simões BJG. Pronto-atendimento de adultos em serviço de saúde universitário: um estudo de avaliação. Rev Adm Pública 1998;32:47-64.

4. Coimbra CEAJr, Santos RV, Yoshida CFY, Baptista ML, Flowers NM, Valle ACF. Hepatitis B epidemiology and cultural practices in ameridian populations of Amazonia: the Tupí-Mondé and the Xavante from Brazil. Soc Sci Med 1996;42:1738-43.

5. Crofts N, Stewart T, Hearne P, Ping XY, Breschkin AM, Locarnini AS. Spread of bloodborne viruses among australian prison entrants. BMJ 1995;310:285-8.

6. D'Amelio R, Matricardi PM, Biselli R, Stroffolini T, Mele A, Spada E et al. Changing epidemiology of hepatitis B in Italy: public health implications. Am J Epidemiol 1992;135:1012-8.

7. Duarte G, Mussi-Pinhata MM, Martinez R, Lemos C, Leite-Figueiredo EM, Quintana SM. Frequência de gestantes portadoras do HBsAg em uma comunidade brasileira. Bol O ficina Sanit Panam 1996;120:189-97.

8. Hadler SC, FAY OH, Pinheiro F, Maynard JE. La hepatitis en las Americas: informe del grupo colaborador de la OPS. Bol O ficina Sanit Panam 1987; 103:185-209.

9. Hosmer DW, Lemeshow S. Applied logistic regression. New York: John Wiley; 1989.

10. Junqueira LAP, Auge APF. Qualidade dos serviços de saúde e satisfação do usuário. Cad FUNDAP 1995;19:60-78.

11. Kiesslich D. Estudo epidemiológico da infecção pelo vírus da hepatite $B$, em doadores de sangue de Manaus, AM - Brasil [tese]. Ribeirão Preto: Faculdade de Medicina de Ribeirão Preto da Universidade de São Paulo; 1996.

12. Mazzur S, Nath N, Fang C, Bastiaans MJ, Molinaris $\mathrm{JL}$, Balcaser $\mathrm{M}$ et al. Distribucion de marcadores de vírus de hepatitis $\mathrm{B}(\mathrm{VHB})$ en la sangre de donadores de 13 países del hemisferio occidental: actas del taller latinoamericano de la Cruz Roja sobre hepatitis B. Bol O ficina Sanit Panam 1980;89:239-48.

13. Ministério da Saúde. Fundação Nacional de Saúde. Guia brasileiro de vigilância epidemiológica. $4^{\mathrm{a}}$ ed Rev. ampl. Brasília: Fundação Nacional de Saúde; 1998.

14. Ministério da Saúde. Portaria $n^{\circ} 1376$ de 19 de novembro de 1993. Normas técnicas para coleta, processamento e transfusão de sangue, componentes e derivados. Diário O ficial da União, Brasília, 1993. seção 1. p. 18405-15.

15. McQuillan GM, Townsend TR, Fields HÁ, Carrol M, Leary M, Polk BF. Seroepidemiology of hepatitis B virus infection in the United States, 1976 to 1980 . Am J Med 1989;87(3A):5-10.

16. Passos ADC, Gomes UA, Figueiredo JFC, Nascimento MMP, Oliveira JM, Gaspar AMC et al. Prevalência de marcadores sorológicos de hepatite B numa pequena comunidade rural do Estado de São Paulo, Brasil. Rev Saúde Pública 1992;26:119-24.

17. Passos ADC, Gomes UA, Figueiredo JFC, Nascimento MMP, Oliveira JM, Gaspar AMC et al. Influência da migração na prevalência de marcadores sorológicos de hepatite B em comunidade rural. 1 - Análise da prevalência segundo o local de nascimento. Rev Saúde Pública 1993;27:30-5. 